\title{
The dynamically disrupted gap in HD 142527
}

\author{
S. Casassus ${ }^{1}$, S. Perez M. ${ }^{1}$, A. Jordán ${ }^{2}$, F. Ménard ${ }^{3,4}$, J. Cuadra $^{2}$, M. R. Schreiber ${ }^{5}$, A. S. \\ Hales $^{6}$, B. Ercolano ${ }^{7}$
}

Received ; accepted

Based on observations obtained at the Gemini Observatory, which is operated by the Association of Universities for Research in Astronomy, Inc., under a cooperative agreement with the NSF on behalf of the Gemini partnership: the National Science Foundation (United States), the Science and Technology Facilities Council (United Kingdom), the National Research Council (Canada), CONICYT (Chile), the Australian Research Council (Australia), Ministério da Ciência e Tecnologia (Brazil) and Ministerio de Ciencia, Tecnología e Innovación Productiva (Argentina). The Gemini run ID is GS-2011A-Q-27. Also based on based on observations made with ESO Telescopes at the Paranal Observatory under DDT program 287.C-5041(A).

\footnotetext{
${ }^{1}$ Departamento de Astronomía, Universidad de Chile

${ }^{2}$ Departamento de Astronomía y Astrofísica, Pontificia Universidad Católica de Chile, 7820436 Macul, Santiago, Chile

${ }^{3}$ UMI-FCA 3386, CNRS / INSU, France and Departamento de Astronomía, Universidad de Chile

${ }^{4}$ Institut de Planétologie et d'Astrophysique de Grenoble (IPAG) CNRS/UJF UMR 5274, Grenoble, France

${ }^{5}$ Departamento de Física y Astronomía, Universidad Valparaiso, Av. Gran Bretana 111, Valparaiso, Chile.

${ }^{6}$ Joint ALMA Observatory, Alonso de Córdova 3107, Vitacura 763-0355, Santiago - Chile

${ }^{7}$ University Observatory, Ludwig-Maximillians University, Munich
} 


\begin{abstract}
The vestiges of planet formation have been observed in debris disks harboring young and massive gaseous giants. The process of giant planet formation is terminated by the dissipation of gas in the protoplanetary disk. The gas-rich disk around HD 142527 features a small inner disk, a large gap from $~ 10$ to 140 AU, and a massive outer disk extending out to $\sim 300$ AU. The gap could have been carved-out by a giant planet. We have imaged the outer regions of this gap using the adaptive-optics camera NICI on Gemini South. Our images reveal that the disk is dynamically perturbed. The outer boundary of the roughly elliptical gap appears to be composed of several segments of spiral arms. The stellar position is offset by $0.17 \pm 0.02^{\prime \prime}$ from the centroid of the cavity, consistent with earlier imaging at coarser resolutions. These transient morphological features are expected in the context of disk evolution in the presence of a perturbing body located inside the cavity. We perform hydro-dynamical simulations of the dynamical clearing of a gap in a disk. A $10 \mathrm{M}_{\text {jup }}$ body in a circular orbit at $r=90 \mathrm{AU}$, perturbs the whole disks, even after thousands of orbits. By then the model disk has an eccentric and irregular cavity, flanked by tightly wound spiral arms, but it is still evolving far from steady state. A particular transient configuration that is a qualitative match to HD 142527 is seen at $1.7 \mathrm{Myr}$.
\end{abstract}

Subject headings: Protoplanetary disks — Planet-disk interactions — Stars: individual 


\section{Introduction}

The lifetime of disks sets the time available for the planet formation process. In the Solar System, chronology of the oldest solid components based on radionuclides (e.g., $\mathrm{Al}^{26}$ ) indicates that the duration timescale of the Solar Nebula was short, of order 2-3 Myr (Montmerle et al. 2006). Direct imaging of a $9 \pm 3 \mathrm{M}_{\text {jup }}$ planet (Lagrange et al. 2010) inside the debris disk of $\beta$ Pic proves that large gas giants can form by $12_{-4}^{+8}$ Myr. In protostellar systems, by 5-10 Myr no gas is left (Pascucci et al. 2006). The evidence points at early giant planet formation, as in the candidate massive protoplanets LkCa 15 b (Kraus \& Ireland) 2012) and T Cha b (Huélamo et al. 2011) (although both remain to be confirmed, e.g. by direct imaging).

Radial gaps in gas-rich protoplanetary disks are currently thought of as possible signposts of planet formation. Previous $H$ and $K$ band coronographic images of HD 142527 reveal a hole in the disk, about 100 AU in radius (Fukagawa et al. 2006). This inner cavity is in fact a gap with an inner boundary, abutting on an inner disk (van Boekel et al. 2004), roughly $10 \mathrm{AU}$ in radius (Anthonioz et al., in preparation), and up to $30 \mathrm{AU}$ (Verhoeff et al. 2011), that accounts for the large near-IR excess of HD 142527 (Fukagawa et al. 2010).

Radiative transfer modelling of the spectral-energy distribution (SED) and NIR images are consistent with an inclination angle of $\sim 20 \mathrm{deg}$ (close to face-on, Verhoeff et al. 2011).

The youth of HD 142527A, at $140 \mathrm{pd} 1$, is evident from the copious amounts of gas (Öberg et al. 2011) in its $\sim 0.1 \mathrm{M}_{\odot}$ circumstellar disk (Verhoeff et al. 2011). Fukagawa et al. (2006) estimate a stellar age of $2_{-1}^{+2} \mathrm{Myr}$ and a mass of $1.9 \pm 0.3 \mathrm{M}_{\odot}$.

Here we report on deep adaptive optics near-IR imaging of the disk and gap of

\footnotetext{
${ }^{1}$ Assuming it is associated to Sco OB2; the Hipparcos parallax of $4.29 \pm 0.98$ marcsec lower-limits its distance to $138 \mathrm{pc}$, at $3 \sigma$.
} 
HD 142527. $\S 2$ describes our observational setups, $\S 3$ emphasizes our main observational

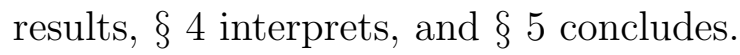

\section{Observations}

NICI adaptive optics imaging. We conducted IR adaptive optics observations of HD 142527 with the NICI coronograph (Biller et al. 2010) on the Gemini telescope. NICI is a dual-channel imager, the images were acquired simultaneously, by pairs, a different filter in each channel. Here we report on two pairs of filters: 1- broadband $K s(2.15 \mu \mathrm{m})$ with $\mathrm{H}_{2}(1-0) \mathrm{S}(1)(2.12 \mu \mathrm{m})$, both with a $0.22^{\prime \prime}$ mask occulting $95 \%$ of the stellar light, and both exposed to $2 \mathrm{~s}$ and $30 \mathrm{~s}$, for a total of $1 \mathrm{~h} ; 2$ - broadband $L^{\prime}(3.8 \mu \mathrm{m})$ with $\operatorname{Br} \gamma(2.17 \mu \mathrm{m})$, both maskless and exposed to $0.38 \mathrm{~s}$, for a total of $1 \mathrm{~h}$. All setups were acquired in stare mode, compensating for parallactic angle rotation. The result of our NICI imaging is shown in Fig. 1, after PSF subtraction 2 .

SINFONI integral field spectra. We also conducted complementary integral-field-unit spectroscopy with the SINFONI instrument on the VLT in order to search for possible planetary objects in the gap. In our setup we chose a very narrow jittering throw that samples the inner $0.45 \times 0.45 \operatorname{arcsec}^{-2}$ only. We processed the ESO pipeline datacubes using spectral deconvolution techniques (Thatte et al. 2007), and also through conventional PSF subtraction (using a PSF standard star). The detection limits we achieve are consistent with those achieved by Thatte et al. (2007) in AB Dor with the same instrument ( 9 mag contrast at $0.2^{\prime \prime}$ separation).

\footnotetext{
${ }^{2}$ we used a Moffat profile fit to the wings of the PSF
} 


\section{Observational results}

\subsection{Size and morphology of the cavity and outer disk inner rim}

The NICI images clearly reveal the non axi-symmetric shape of the cavity. This is the highlight of our observations. The shape is roughly elliptical, albeit with significant

departures, with a representative eccentricity of $e_{c}=\sqrt{1-\left(b_{c} / a_{c}\right)^{2}} \approx 0.5$ (where $a_{c}$ and $b_{c}$ are the ellipse axes). But, as illustrated in Fig. 2, the exterior ring can be described as being composed of four arms overlapping in their extremities, except for two small, $\sim 0.2$ arcsec gaps or intensity nulls along the ring, due north and south-south-east from the star. The observation of these morphological feature is based on the shape of the inner rim of the ring seen in direct images (even without subtraction of the PSF glare). Additionally, an unresolved brightness increment, or knot, can be seen along the north-western segment (which is also the brightest segment) - the alignment of this unresolved feature in all filters is illustrated in the RGB image, although we cannot determine from these data alone if this knot is real or perhaps only a ghost or a chance alignment of speckles.

The south-western spiral arm segment (labelled arm 2 on Fig. 2) sprouts away from the outer ring at a PA of $-45 \mathrm{deg}$ (East of North), where it extends into the outer arm seen by Fukagawa et al. (2006). This outer arm is at much fainter levels than the outer ring - our dataset has finer resolution but is shallower, so that only its root is seen in our figures. As discussed in Fukagawa et al. (2006), this outer arm could have been triggered by a stellar encounter, but the putative partner remains to be identified. A nearby point source turned out to be a background star (Fukagawa et al. 2010) - in our NICI images this source is located at a separation of 5."44 from HD 142527, and a PA of 220 deg (East of North).

The inner radius of the ring varies from $0.7^{\prime \prime}$ to the north, to about $1.0^{\prime \prime}$ to the south, and a projection effect is discarded since the central star is clearly offset by $0.17 \pm 0.02^{\prime \prime}$ 
from the centre of this approximate ellipse. This offset was first noted by Fukagawa et al. (2006), albeit at lower angular resolution.

In $K$-band the west side of the rim we see is brighter, in agreement with

Fukagawa et al. (2006). Fujiwara et al. (2006) and Verhoeff et al. (2011) already noted that in the thermal IR, $N$ and $Q$ bands, it is the eastern side that is brightest, which is consistent with a projection effect (Fujiwara et al. 2006). At the shorter wavelength $K$-band, the front (i.e. nearest) side of the disk appears brighter because of forward scattering at the surface of the disk. On the opposite, the thermal emission from the larger projected surface of the rim of the back side of the disk appears brighter than the slimmer (in projection) rim of the near side. This implies a major axis of the disk in the North-South direction and a minor axis in the E-W direction (as observed here). The emission null we detect due North is seen on both sets of Subaru images, at $K$-band and in the mid-IR as well.

\subsection{Detection limits in the range 14-35 AU}

No companion is clearly detected in our NICI images. An upper limit on a binary companion is difficult to obtain because of the speckle noise, and because of the lack of accurate NICI zero points. However, the SINFONI observations allow us to place a lower limit flux ratio at $0.2-0.3^{\prime \prime}$ separation of 1905 at $3 \sigma$ in $K$; Fig. 3 illustrates this upper limit. Modeling the SED (with MCFOST, Pinte et al. 2006) we estimate that half of the total flux density of HD 142527 in K can be assigned to the disk. In that case, the SINFONI flux ratio limit of 1905 corresponds to an absolute magnitude $M_{K}>8.2$ at $3 \sigma$. As a comparison point we refer to the COND03 tracks (Baraffe et al. 2003) at 1 Myr of age, which would imply a mass of less than $12 \mathrm{M}_{\text {jup }}$ given this $M_{K}$ limit. However the age of HD 142527 and the models are not precise enough to ascertain this upper limit mass value. We stress again that this limit constrains the presence of massive gaseous giants at stellocentric radii of 14 
to $35 \mathrm{AU}$.

\section{Analysis}

\subsection{Arguments in favor of a planet-created gap for HD 142527}

The contribution from photoevaporation to cavity clearing can be neglected in this case. The inner disk, the large gap and the vigorous accretion rate (Garcia Lopez et al. 2006) of $7 \times 10^{-8} \mathrm{M}_{\odot} \mathrm{yr}^{-1}$ argue against photoevaporation. An X-ray driven wind can indeed explain a subset of the observed accreting disks with inner holes, but only those with radii smaller than $20 \mathrm{AU}$ and accretion rates less than $10^{-8} \mathrm{M}_{\odot} \mathrm{yr}^{-1}$ (Owen et al. 2011).

Gap clearing by single or multiple planets could potentially explain wide gaps observed in transitional disks (Rice et al. 2003). We thus focus on the simplest possible scenario to interpret the morphology of the cavity in HD 142527's disk, i.e. dynamical clearing by one single embedded and massive-Jupiter-size planet opening a gap in its gaseous disk. The maximum gap opening width will depend on the mass and the orbital parameters of this planet (Dodson-Robinson \& Salvk 2011). The cavity forms due to the torque exerted by the star-planet binary (Artymowicz \& Lubow 1994). Elliptical cavities have been predicted with $e_{\mathrm{c}} \approx 0.25$ in the case of circular binaries with mass ratios $>3 \times 10^{-3}$, in which case the gap becomes large enough to deplete the region in which the eccentricity-damping outer 1:3 Lindblad resonance is produced (Kley \& Dirksen 2006). Further numerical work (Hosseinbor et al. 2007) shows that, for eccentric binaries, the outer edge of the cavity becomes even more eccentric. The observed eccentric cavity is then a recurrent feature of gap-clearing models. 


\subsection{Fargo hydrodynamic simulations of gap clearing by a planet}

In order to test further the single-planet origin for the wide gap in the gaseous disk of HD 142527, we conducted hydrodynamic simulations with FARGO. Our goal here is not to fit the data but to verify qualitatively the tenability of this scenario for HD 142527. FARGO is a dedicated grid-based 2D code publicly available on the web (Masset 2000), and specifically designed for planet-disk interactions. The run presented in this paper corresponds to a system evolution equivalent to $1.7 \mathrm{Myr}$.

We picked common fiducial parameters for the model, as detailed below. The model disk is initially axisymmetric, its surface density is initially a power law of radius, $\Sigma=\Sigma_{0}\left(r / r_{0}\right)^{-1}$, where $\Sigma_{0}=566 \mathrm{~g} \mathrm{~cm}^{-2}\left(6.3 \times 10^{-5} \mathrm{M}_{\odot} \mathrm{AU}^{-2}\right)$ at $r_{0}=1 \mathrm{AU}$, so slightly smaller than the value assumed by Dodson-Robinson \& Salyk (2011). The model disk has a constant pressure scale height $H$ over radius $r$ with value $H / r=0.05$. For the disk calculations the boundaries are 4 and 300 AU for the inner and outer radii, respectively, giving a total mass of $0.1 \mathrm{M}_{\odot}$. The inner boundary is open, allowing mass to fall onto the star, while the outer boundary is such that we allow for a steady state mass transfer into the disk. Accretion across the disk is modeled by using an $\alpha$ prescription (Shakura \& Sunyaev 1973). We adopted $\alpha=0.002$ following Dodson-Robinson \& Salyk (2011). From the SINFONI limits described above we know that any planet present in the inner 14 to $35 \mathrm{AU}$ cannot have a mass exceeding $\sim 12$ Jupiter masses. We assume no such planet is present further out in the disk either, and chose a planet mass of $10 M_{\mathrm{Jup}}$.

We placed a $10 M_{\text {Jup }}$ planet in a fixed circular orbit at $90 \mathrm{AU}$ in the model disk. This radius was chosen so that the planet's sphere of influence, given by a few times its Hill radius (e.g. Dodson-Robinson \& Salyk 2011), reaches the observed gap size of 130 AU. Migration was inhibited until a sizeable gap was carved by the planet. This artificial constraint stems from the lack of heat or momentum diffusion in FARGO (Dodson-Robinson \& Salyk 2011). 
The resulting gas surface density field is shown in Fig. 4. This specific dynamically perturbed morphology will persist over a timescale of a few tens of thousand years (i.e, around 100 orbits) but is not in steady state. The rim will remain asymmetric over time, and is composed of tightly wound spiral arms, whose superposition forms a roughly elliptical cavity. The cavity centroid is persistently offset from the star, but any particular morphology of the gap is transient and lasts about 100 orbits. In this snapshot view (fig. 44), the outer edges of the cavity are most reminiscent of those seen in the NICI images. The cavity eccentricity is $\sim 0.6$, and its centroid is offset from the star by $\sim 16$ AU.

As seen in Fig. 4, this model produces a massive inner disc, extending out to 50-60 AU in radius, which is not consistent with the observations. Additional ingredients are required to deplete that region in the framework of a dynamical clearing model, e.g., additional planets closer to the star (as in Dodson-Robinson \& Salyk 2011). Fitting the inner disc is left for future work. We stress that the purpose of these simulations is to provide a plausible explanation of the NICI observations of the inner edge of the outer disk only.

\section{Summary and discussion}

Our observations of HD 142527 indicate that the inner rim of its outer disk is roughly elliptical, and composed of an overlapping set of spiral arm segments. We confirm that the star is offset by $0.17 \pm 0.02^{\prime \prime}$ from the centroid of the cavity. No companion is detected in the range of 14 to $35 \mathrm{AU}$, with a lower limit $M_{K}>8.2$ at $3 \sigma$ (for a distance of $140 \mathrm{pc}$ ).

The observed morphology of the disk in HD 142527 suggests a dynamically perturbed state. The segmented spirals, and the offset between the cavity centroid and the star, can be seen among the varied morphologies predicted by hydrodynamic simulations of HD 142527. To explore, at least qualitatively, the origin of the cavity and its peculiar shape, 
we reported on a simple case. A massive protoplanet on a circular orbit whose radius is comparable to the disk's outer edge perturbs the entire disk, which does not reach steady state even after thousands of orbits. The global shape of the cavity is a long-standing feature; the general asymmetries (off-center star, eccentricity of the cavity) are imprinted early and remain for the duration of the calculations. However, the specific configuration of the model run, position of the streamers, and position and number of spiral arms, form a specific morphology that is only a transient phase. A particular morphology may recur, but lasts for a limited number of orbits. That phase which qualitatively matches HD 142527 lasts for a hundred orbits or so.

It is tempting to suggest that the observed shape of the disk rim of HD 142527 today will also be brief compared to its age, with features that are unlikely to be found in other systems that are also undergoing dynamical clearing. In this scenario, snapshot imaging of transition disks are likely to look different in their fine details. However, there should be common features like off-centering of the central star, non axi-symmetry of the disk rim, and presence of spiral arms. Current imaging campaigns are indeed revealing these structures, as in HD 100546 (Grady et al. 2001); HD 135344B (Muto et al. 2012); AB Aur (Hashimoto et al. 2011). Interstingly, the list of known transition disks is rapdily increasing with improving observational capabilities both in direct imaging at optical/near-IR (see e.g., the citations above) and in the sub-mm regime (Andrews et al. 2011). We should be able to verify these ideas in the near future, specifically that the observed morphological variety of transition disks derives from a common dynamical history in this evolutionnary stage when there is large-scale feedback between the planet formation process and its parent disk.

A key to test this transient scenario for HD 142527 and transition disks in general would be to detect the gap-crossing planetary accretion flow and link it to the outer-disk 
asymmetries. Meanwhile, a quantitative comparison with hydrodynamic models requires radiative transfer calculations on the model density fields.

While this article was under review Biller et al. (2012) reported on a tentative detection of a low-mass stellar companion to HD 142527, at 13 AU, so abutting on the inner disk. This companion is inferred through parametrised-modeling of visibility data. If real, this stellar companion will strongly impact on the inner disk, and its inclusion in simulation may bring closer agreement with the observed inner disk properties. This would be particularly interesting given that our one-planet scenario currently fails to reproduce the properties of the inner disk. It is currently too large in radius and mass, compared with observations. Including a second body in the simulation would produce a larger gap with a smaller inner disk (e.g, Dodson-Robinson \& Salyk 2011). However, HD 142527B alone probably cannot explain the extent of the cavity. A single body cannot clear a gap wider than $\sim 5$ times its tidal radius (e.g. Dodson-Robinson \& Salyk 2011), given by $R_{\text {hill, where }}$ $R_{\text {hill }}=a\left(M_{p} / 3 M_{\star}\right)^{1 / 3}$ is known as the Hill radius. Mass and semi-major axis estimates for the companion star detected by Biller et al. (2012) give a Hill radius $R_{\text {hill }} \sim 3-5$ AU.

SC, SP, AJ, FM, MS and AH acknowledge support from the Millennium Science Initiative (Chilean Ministry of Economy), through grant "Nucleus P10-022-F". We thank the ESO Director General for discretionary time that allowed the K-band limit. SC, AJ and JC thank the CATA ("Fondo Basal PFB-06, CONICYT"). SC acknowledges FONDECYT grant 1100221. AJ acknowledges additional support from Anillo ACT-086 and FONDECYT project 1095213. FM acknowledges support from PNPS of CNRS/INSU and from EU FP7-2011 under grant agreement No. 284405 (DIANA). JC acknowledges VRI-PUC (Inicio 16/2010) and FONDECYT 11100240. 

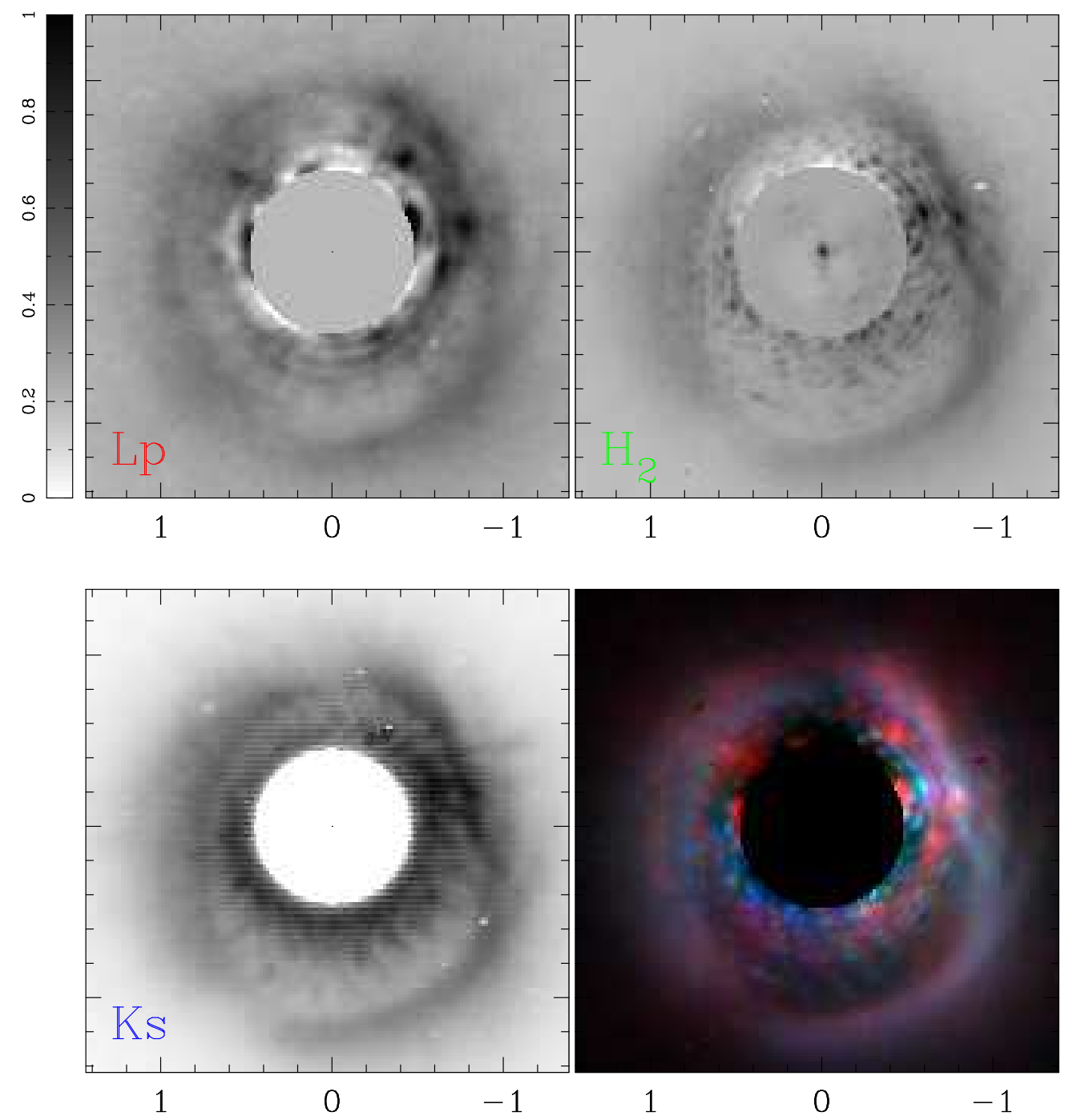

Fig. 1.- Infrared adaptive-optics imaging of HD142527 and its protoplanetary disks, from 11-Jun-2011. In x and y we indicate offset J2000 RA \& Dec, in arcsec (so North is up and East is to the left). From left to right we show reduced images obtained in $L_{p}, \mathrm{H}_{2}(1-0) \mathrm{S}(1)$, and $K_{s}$, and an RGB combination following the color codes indicated in each image. The linear gray scales are normalised over the entire range of intensity values outside a radius of 0.45 arcsec, containing the core of the diffracted stellar light, and abutting on the halo of the stellar glare. Inside this region the images are masked, except for the $\mathrm{H}_{2}$ image, where the stellar PSF has been attenuated with azimuthal averages. A roughly elliptical outer ring is clearly seen. The star is offset from the center of the cavity by $0.17 \pm 0.02$ arcsec. 


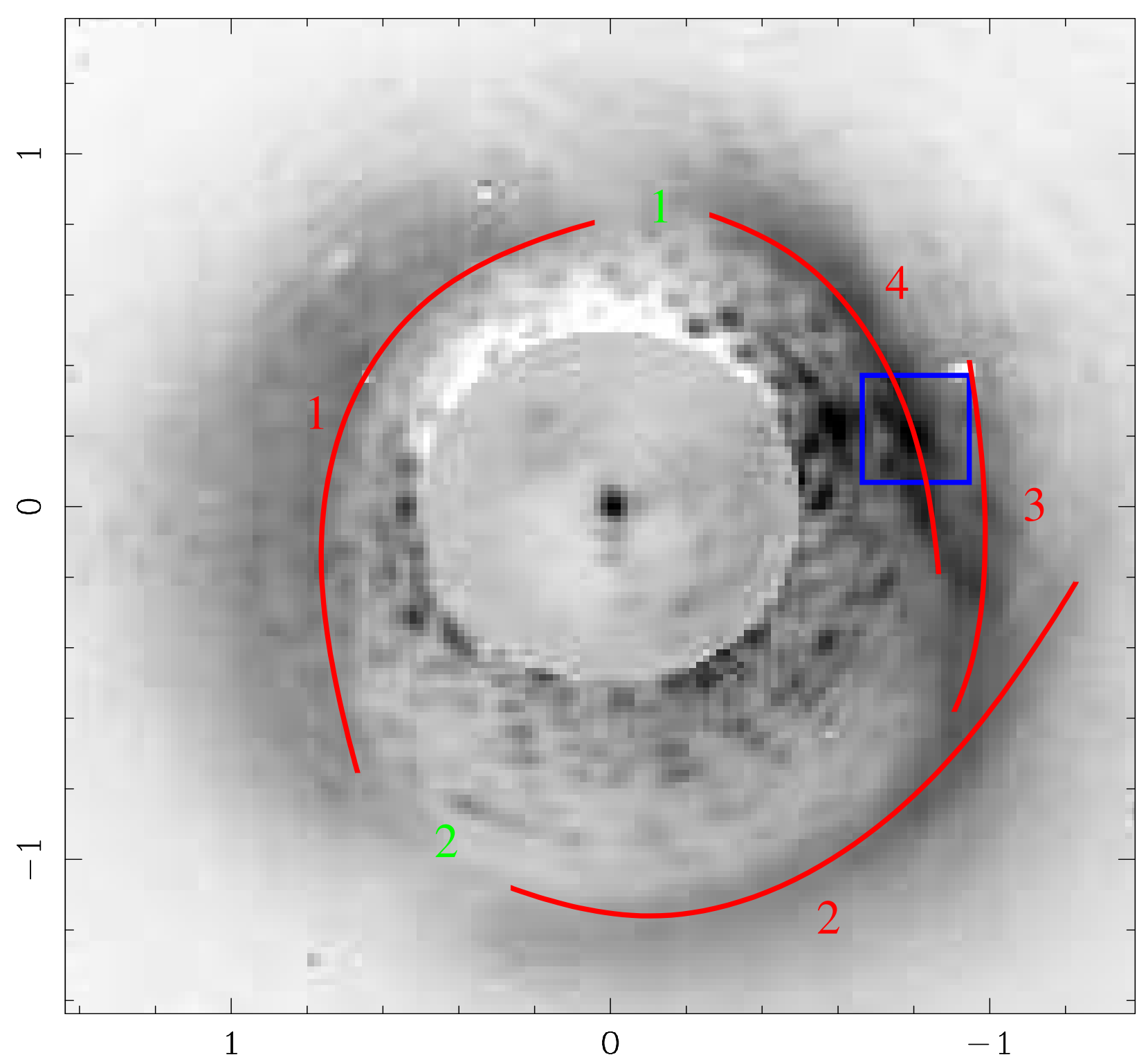

Fig. 2.- Annotations on the $\mathrm{H}_{2}(1-0) \mathrm{S}(1)$ image also show on Fig. 1. In red we indicate the spiral arms we could identify, with red identification numbers. The green numbers indicate the positions of the two intensity nulls, or gaps, along the ring. The blue box surrounds the position of the knot. 


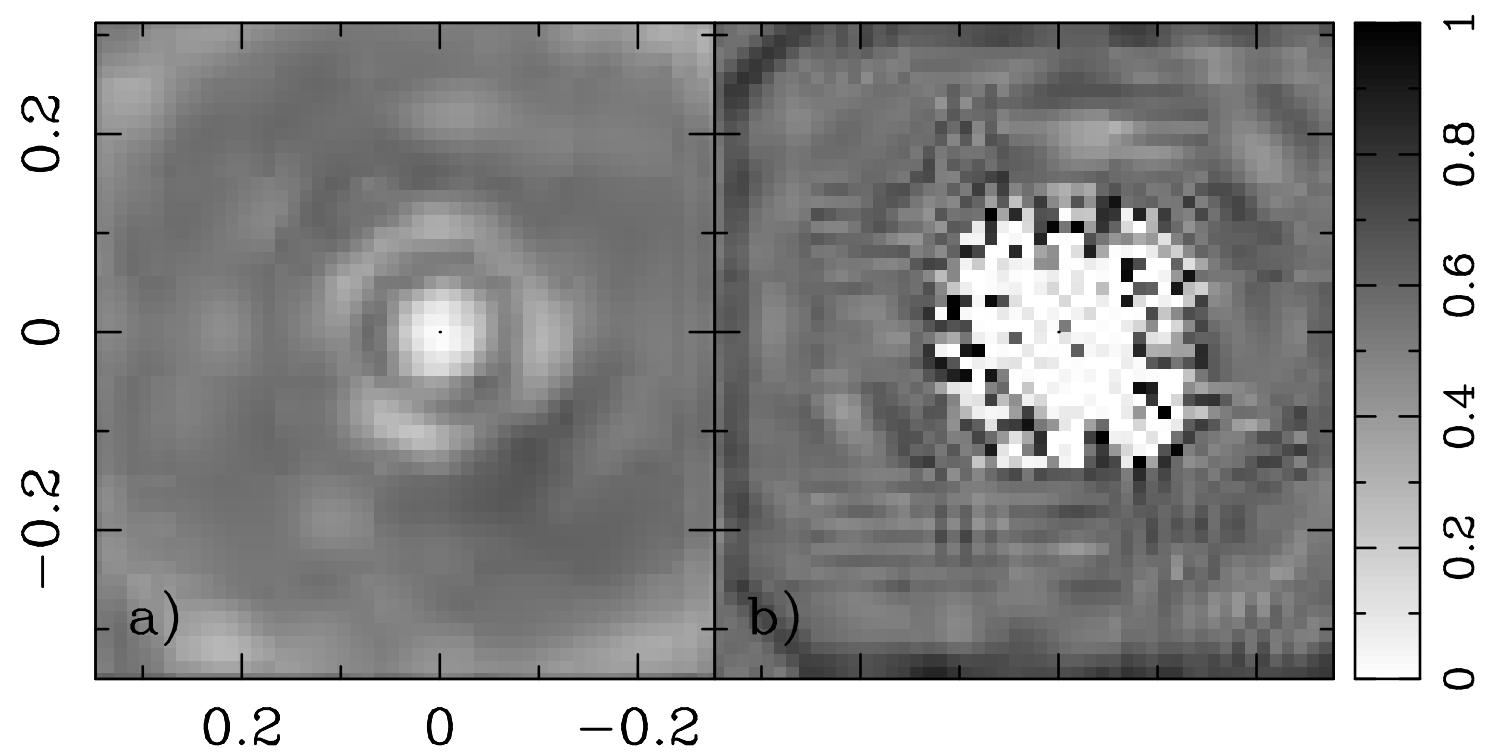

Fig. 3.- Upper limits on the $K_{s}$ magnitude of HD 142527b. Axis labels are as for Fig. 1. In a) (left panel) we show the collapsed SINFONI datacube, as produced by the pipeline, but divided by an azimuthal average chosen to highlight the PSF pattern. In b) (right panel) we show the collapsed image of the spectrally-deconvolved and PSF-subtracted data cube, with a linear intensity scale streching from -0.75 to 0.57 , in counts, where the maximum number of counts in the collapsed cube (shown in a)) is 548. The root-mean-square dispersion of the counts in an annulus from 0.2 to 0.3 arcsec is $9.610^{-2}$, or a $3 \sigma$ lower limit to the flux ratio of 1905. Using a separate PSF standard, this residual rms value is 0.19 , giving a $3 \sigma$ lower limit to the flux ratio of 966 . 


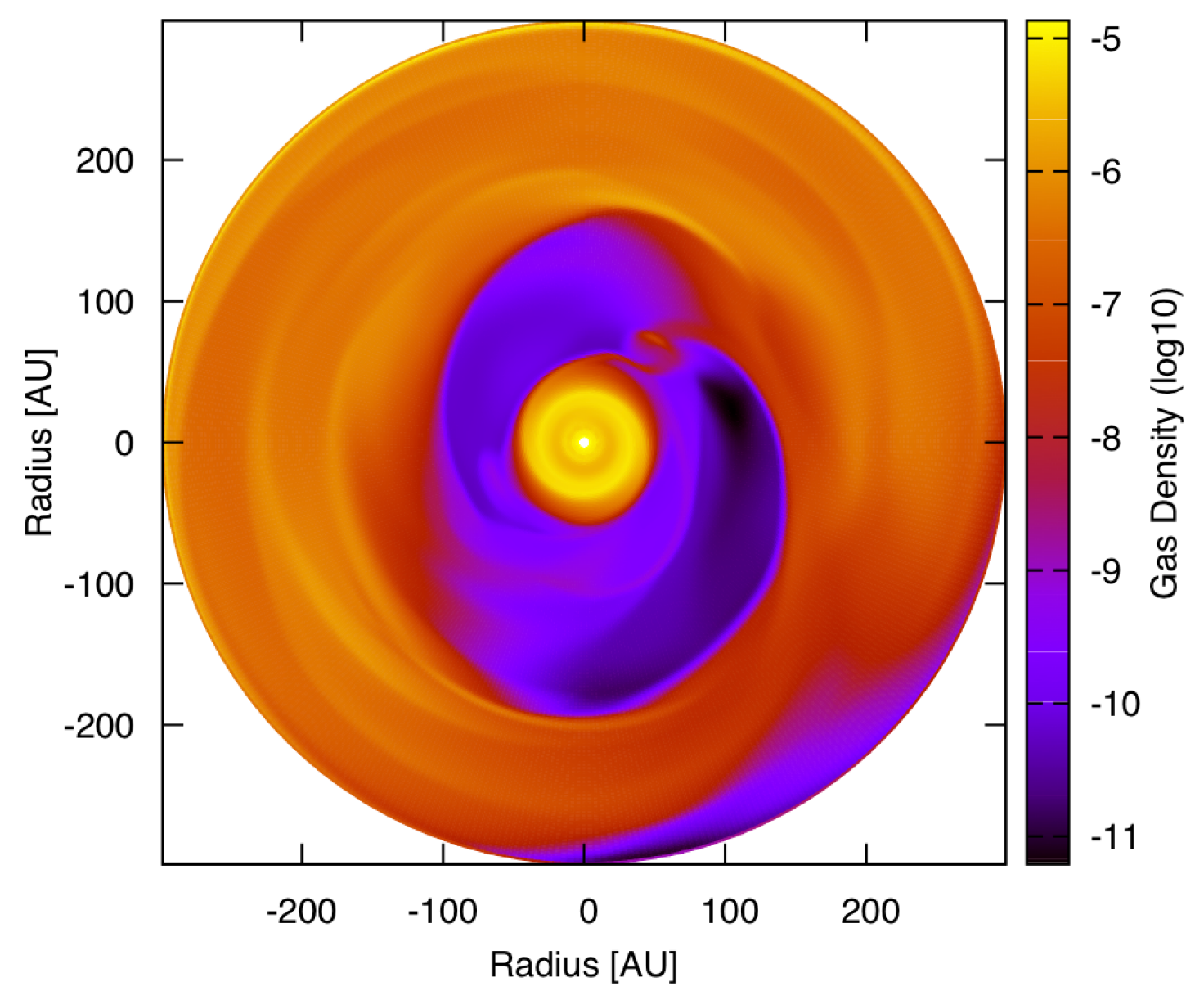

Fig. 4.- FARGO simulation of a $10 \mathrm{M}_{\text {jup }}$ planet embedded in a $0.1 \mathrm{M}_{\odot}$ disk. The spatial axes are in AU. The color scale represents the surface density in a logarithmic ramp in FARGO code units in which $10^{-5}$ equals $88 \mathrm{~g} \mathrm{~cm}^{-2}$. Notice the stellar shift relative to the position of the cavity centroid of $\sim 16 \mathrm{AU}$. 


\section{REFERENCES}

Andrews, S. M., Wilner, D. J., Espaillat, C., Hughes, A. M., Dullemond, C. P., McClure, M. K., Qi, C., \& Brown, J. M. 2011, ApJ, 732, 42

Artymowicz, P., \& Lubow, S. H. 1994, The Astrophysical Journal, 421, 651

Baraffe, I., Chabrier, G., Barman, T. S., Allard, F., \& Hauschildt, P. H. 2003, A\&A, 402, 701

Biller, B., et al. 2012, ArXiv e-prints

Biller, B. A., et al. 2010, ApJ, 720, L82

Dodson-Robinson, S. E., \& Salyk, C. 2011, ApJ, 738, 131

Fujiwara, H., et al. 2006, ApJ, 644, L133

Fukagawa, M., Tamura, M., Itoh, Y., Kudo, T., Imaeda, Y., Oasa, Y., Hayashi, S. S., \& Hayashi, M. 2006, ApJ, 636, L153

Fukagawa, M., et al. 2010, PASJ, 62, 347

Garcia Lopez, R., Natta, A., Testi, L., \& Habart, E. 2006, A\&A, 459, 837

Grady, C. A., et al. 2001, AJ, 122, 3396

Hashimoto, J., et al. 2011, ApJ, 729, L17

Hosseinbor, A. P., Edgar, R. G., Quillen, A. C., \& LaPage, A. 2007, Monthly Notices of the Royal Astronomical Society, 378, 966

Huélamo, N., Lacour, S., Tuthill, P., Ireland, M., Kraus, A., \& Chauvin, G. 2011, A\&A, $528, \mathrm{~L} 7+$ 
Kley, W., \& Dirksen, G. 2006, A\&A, 447, 369

Kraus, A. L., \& Ireland, M. J. 2012, ApJ, 745, 5

Lagrange, A.-M., et al. 2010, Science, 329, 57

Masset, F. 2000, A\&AS, 141, 165

Montmerle, T., Augereau, J.-C., Chaussidon, M., Gounelle, M., Marty, B., \& Morbidelli, A. 2006, Earth Moon and Planets, 98, 39

Muto, T., et al. 2012, ApJ, 748, L22

Öberg, K. I., et al. 2011, ApJ, 734, 98

Owen, J. E., Ercolano, B., \& Clarke, C. J. 2011, MNRAS, 412, 13

Pascucci, I., et al. 2006, ApJ, 651, 1177

Pinte, C., Ménard, F., Duchêne, G., \& Bastien, P. 2006, A\&A, 459, 797

Rice, W. K. M., Wood, K., Armitage, P. J., Whitney, B. A., \& Bjorkman, J. E. 2003, MNRAS, 342, 79

Shakura, N. I., \& Sunyaev, R. A. 1973, A\&A, 24, 337

Thatte, N., Abuter, R., Tecza, M., Nielsen, E. L., Clarke, F. J., \& Close, L. M. 2007, MNRAS, 378, 1229

van Boekel, R., et al. 2004, Nature, 432, 479

Verhoeff, A. P., et al. 2011, A\&A, 528, A91+ 